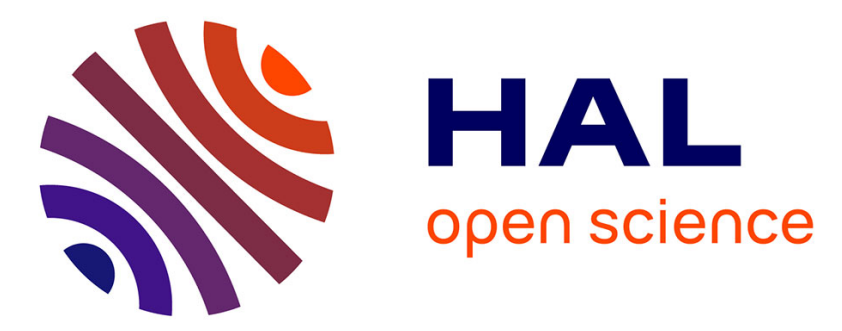

\title{
Sur l'utilisation du bêta-carotène pour la normalisation de la couleur du beurre
}

\author{
J. Casalis, Y. Chardon, F. M. Luquet, P. Mainguy, B. Watier, M. Yver
}

\section{To cite this version:}

J. Casalis, Y. Chardon, F. M. Luquet, P. Mainguy, B. Watier, et al.. Sur l'utilisation du bêta-carotène pour la normalisation de la couleur du beurre. Le Lait, 1972, 52 (513_514), pp.220-228. hal-00928578

\section{HAL Id: hal-00928578 \\ https://hal.science/hal-00928578}

Submitted on 1 Jan 1972

HAL is a multi-disciplinary open access archive for the deposit and dissemination of scientific research documents, whether they are published or not. The documents may come from teaching and research institutions in France or abroad, or from public or private research centers.
L'archive ouverte pluridisciplinaire HAL, est destinée au dépôt et à la diffusion de documents scientifiques de niveau recherche, publiés ou non, émanant des établissements d'enseignement et de recherche français ou étrangers, des laboratoires publics ou privés. 


\title{
Sur l'utilisation du bêta-carotène pour la normalisation de la couleur du beurre
}

\author{
par \\ J. CASALIS*, Y. CHARDON*, F. M. LUQUET**, P. MAINGUY***, \\ B. WATIER ${ }^{* * *}$ et M. YVER***
}

\section{Introduction}

I. - Les correctifs de coloration actuellement utilisés.

II. - Intérêt de l'utilisation du ß-carotène pour normaliser la couleur du beurre.

III. - Préparation de l'émulsion de $\beta$-carotène.

$I V$. - Utilisation de l'émulsion de $\beta$-carotène.

$V$. - Méthodologie de la normalisation.

Conclusion.

\section{Introduction}

Dans une précédente publication (1), nous avons précisé d'une part l'importance des variations actuelles du taux de $\beta$-carotène dans le lait, et leurs différentes causes (climatiques, géographiques, alimentaires...) et d'autre part l'étroite corrélation qui existe entre les mesures trichromatiques de la couleur du beurre et sa teneur en $\beta$-carotène.

Les enquêtes qui ont ensuite été effectuées ont montré que la coloration naturelle des beurres français variait du blanc pâle au jaune soutenu, et que ceci correspondait à un assez large éventail de teneur en $\beta$-carotène (depuis la présence de traces, jusqu'à 10 et même $12 \mathrm{ppm}$ ). En tenant compte des variations saisonnières et géographiques la teneur annuelle moyenne pondérée du beurre français est de 3 à $4 \mathrm{ppm}$ de $\beta$-carotène. Or la couleur est un des critères

* ENSIA, Douai.

** LRIL, Douai.

*** HOFFMANN-LA ROCHE, Neuilly.

(1) J. CASALIS et al., Le Lait, $\mathrm{n}^{\circ} 511-512$, p. 28. 
de choix du consommateur, et, si l'on estime que sur l'ensemble du marché français, la couleur souhaitée par le consommateur correspond à une teneur moyenne de $7 \mathrm{ppm}$ de $\beta$-carotène, on voit que, dans le but de satisfaire ce désir, les fabricants devront effectuer une normalisation de la couleur de leurs beurres par une complémentation en pigment, lorsque celle-ci s'avère nécessaire.

\section{I. - Les correctifs de coloration actuellement utilisés}

Le report d'une saison à l'autre de crèmes soumises à une congélation préalable, pratiqué dans certaines beurreries françaises, pourrait être envisagé comme un moyen d'effectuer une correction de couleur. Mais il s'agit là d'une opération coûteuse, et la conservation des crèmes congelées n'est pas sans présenter des risques sérieux, tant au point de vue de la technologie de fabrication, qu'à celui des qualités organoleptiques des produits fabriqués.

L'industrie laitière utilise depuis longtemps un colorant végétal naturel, le rocou, extrait des graines du rocouyer (Bixa orellana) arbrisseau exotique cultivé en Amérique du Sud, en Guyane et surtout aux Indes.

Les extraits commerciaux sont des mélanges complexes, en suspension huileuse, de différents constituants dont les pigments principaux sont la bixine et la norbixine, qui ne possèdent aucune valeur provitaminique.

La couleur obtenue est voisine de celle donnée naturellement ou artificiellement par le $\beta$-carotène mais l'inconvénient du rocou est qu'il provient de graines dont la teneur en pigments est très variable et que les extraits obtenus présentent des différences considérables de pouvoir colorant.

Des contrôles effectués au laboratoire sur des solutions commerciales de rocou ont mis en évidence des teneurs allant de 0,18 à 0,34 p. 100 en équivalent $\beta$-carotène. Ceci est un obstacle majeur à une complémentation sûre et facilement calculable.

\section{II. - Intérêt de l'utilisation du $\beta$-carotène pour normaliser la couleur du beurre}

L'hétérogénéité de la couleur du beurre constatée sur le marché français montre que l'utilisation du rocou n'a pas apporté à l'industrie beurrière une solution efficace. Une nouvelle possibilité est offerıe par l'utilisation du $\beta$-carotène de synthèse. Le $\beta$-carotène est un constituant naturel de beaucoup d'aliments et en particulier du lait et du beurre. Lors de son utilisation par l'organisme, il est transformé en vitamine A (fig. 1) et présente toutes garanties sur les plans nutritionnel et toxicologique. En particulier son innocuité a été parfaitement démontrée : à la suite d'études approfondies, les experts de la commission mixte FAO-OMS sur les additifs alimentaires l'ont 
admis dans la classe $\mathrm{A}$, où sont rangées les substances présentant les meilleures garanties d'innocuité.

La synthèse du $\beta$-carotène a été réalisée en 1950 par Isler. On l'obtient à l'heure actuelle à l'état chimiquement pur. Ainsi peut-on mettre à la disposition de l'Industrie laitière un produit identique au pigment naturel du lait, et dont la teneur en principe colorant est normalisée et constante. L'adjonction de cette substance a l'avantage

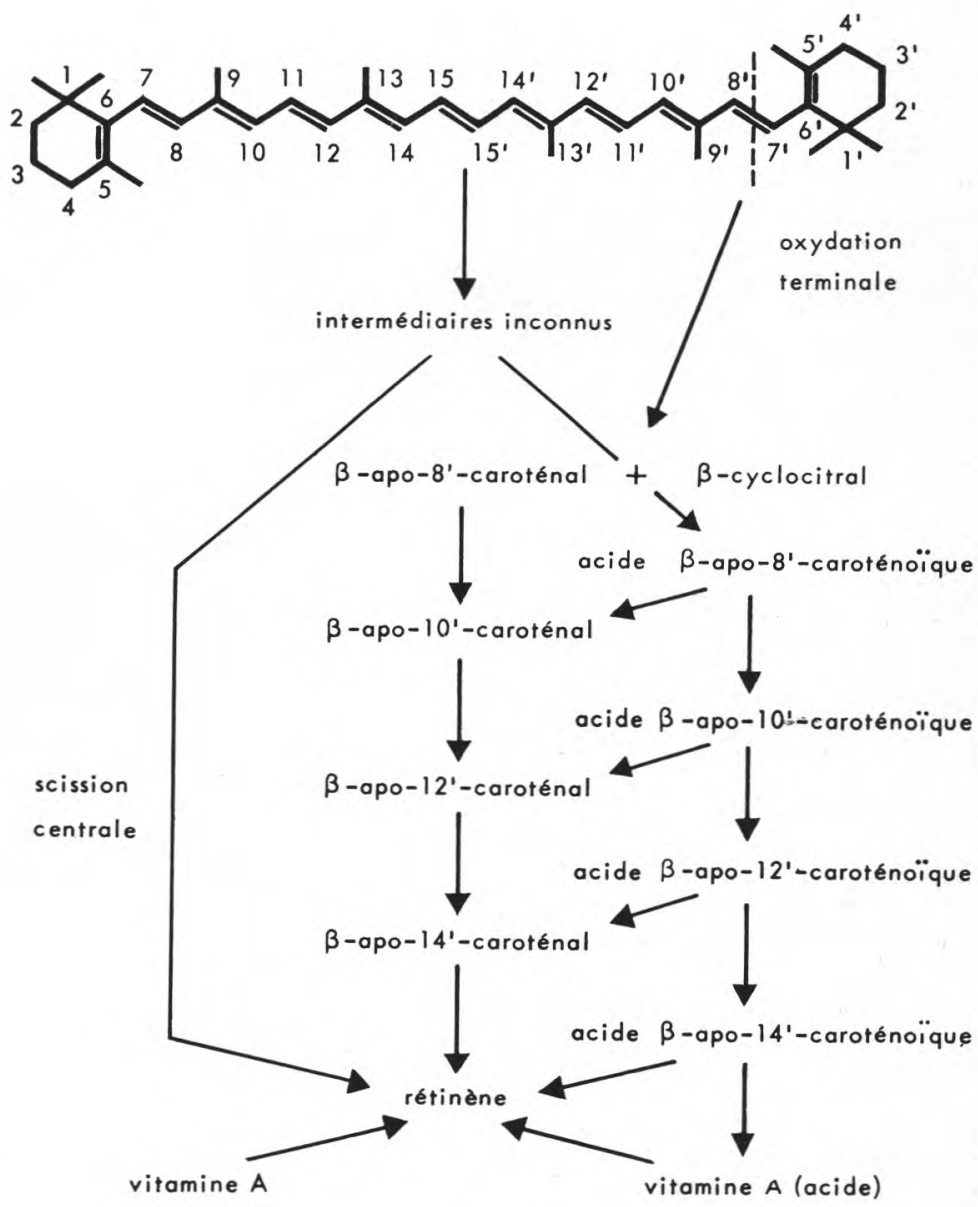

fig. 1

Schéma de la dégradation oxydative du $\beta$-carotène, selon J. Glover 
de ne pas affecter la composition qualitative du beurre et doit donc retenir l'attention de l'hygiéniste et du nutritionniste.

\section{III. - Mise au point d'une forme de $\beta$-carotène adaptée à l'industrie laitière}

Le $\beta$-carotène de synthèse est inutilisable à l'état pur pour colorer le beurre, car il ne se dissout pas dans la crème. Il a donc fallu rechercher une formule spécialement adaptée aux techniques beurrières, et en particulier à la fabrication continue du beurre. Après de nombreuses recherches, une émulsion huile dans eau contenant 2 p. 100 de $\beta$-carotène a été mise au point (1).

Des contrôles effectués au laboratoire ont mis en évidence la stabilité de cette émulsion, tant au point de vue physique qu'au point de vue chimique. Ces études ne sont pas terminées mais elles ont permis de montrer qu'après 4 mois de stockage à une température de $+5^{\circ} \mathrm{C}$, aucune modification n'est apparue et qu'en particulier la teneur en $\beta$-carotène de l'émulsion est restée constante.

TABLEAU 1

Essai de conservation de l'émulsion à $+5^{\circ} \mathrm{C}$

\begin{tabular}{c|c|c|c|c|c}
\hline Temps & 0 & 1 mois & 2 mois & 3 mois & 4 mois \\
\cline { 2 - 3 } Teneur en p. 100 & 2,2 & 2,2 & 2,2 & 2,2 & 2,2 \\
\cline { 2 - 4 } & & & & & \\
\hline
\end{tabular}

\section{IV. - Utilisation de l'émulsion de $\beta$-carotène}

\section{ESSAI DE LABORATOIRE}

Un certain nombre de déterminations trichromatiques ont été effectuées sur des beurres prélevés dans le commerce d'une part, sur des beurres préparés au laboratoire d'autre part. Elles ont permis de constater qu'il existe une relation linéaire entre les coefficients $\mathrm{x}$ et $\mathrm{y}$, qu'il s'agisse d'échantillons prélevés dans le commerce ou préparés au laboratoire. Le coefficient de corrélation est de 0,993 ce qui est très satisfaisant, compte tenu des imprécisions dues aux manipulations lors des déterminations (fig. 2).

(1) Il s'agit d'un produit contenant environ $30 \mathrm{p} .100$ d'huile végétale. Au taux d'incorporation considéré, l'apport de matière grasse étrangère est inférieur à $100 \mathrm{ppm}$. 


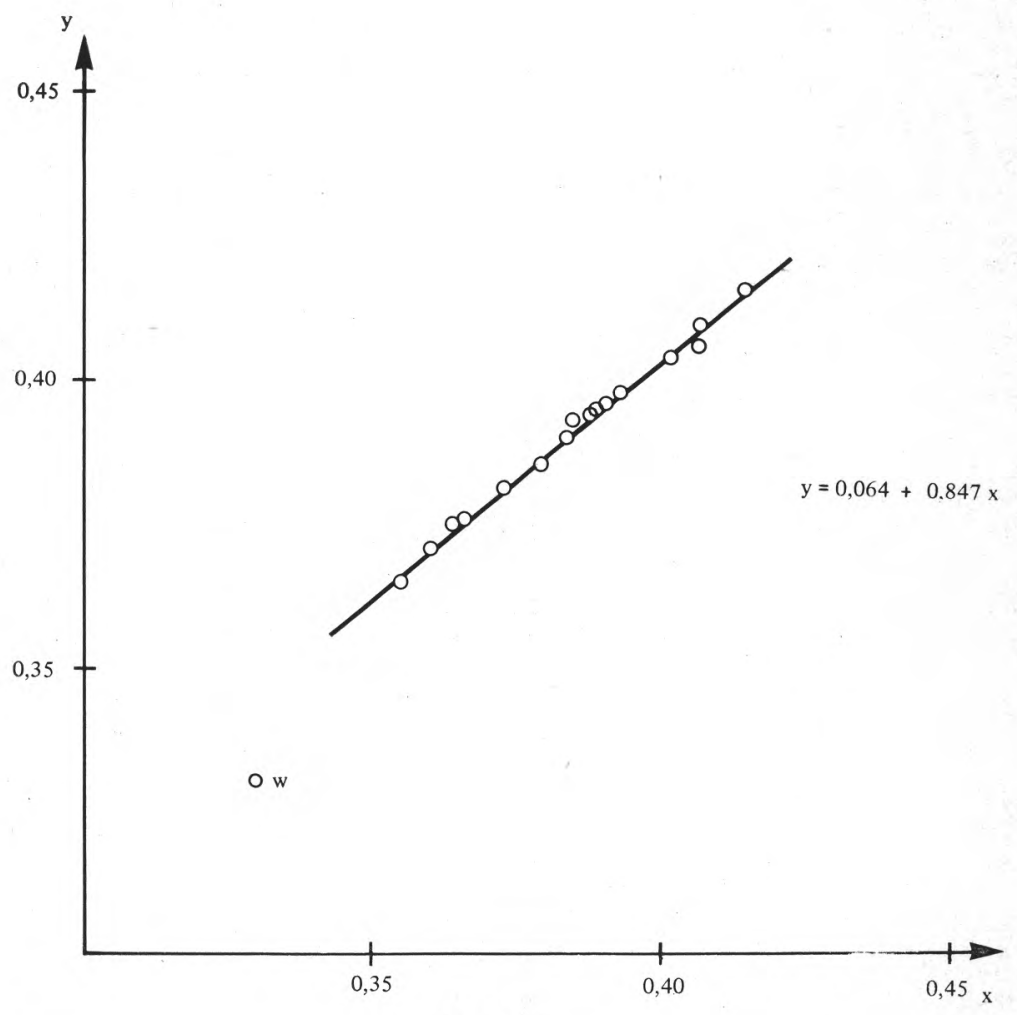

fig. 2

Etude de la corrélation entre $\mathrm{x}$ et $\mathrm{y}$

TABLEAU 2. - Essais de laboratoire

\begin{tabular}{l|c|c|c|c|c}
\hline \multicolumn{1}{c|}{ Echantillon } & 1 & 2 & 3 & 4 & 5 \\
\cline { 2 - 3 } & & & & & \\
Teneur ajoutée* & 0,128 & 0,245 & 0,371 & 0,572 & 1,100 \\
Teneur théorique* & 0,270 & 0,369 & 0,490 & 0,680 & 1,220 \\
Teneur trouvée* & 0,293 & 0,380 & 0,520 & 0,660 & 1,185 \\
Rendement théorique & 108 p. 100 & 103 p. 100 & 105 p. 100 & 97 p. 100 & 97 p. 100 \\
\hline
\end{tabular}

* En mg de $\beta$-carotène p. $100 \mathrm{~g}$ de beurre. 
Par ailleurs, des contrôles effectués sur la teneur en $\beta$-carotène des crèmes, de l'émulsion et des beurres obtenus ont permis de constater que la totalité du $\beta$-carotène passe dans la phase grasse du produit fini (tab. 2).

\section{ESSAI EN FABRICATION INDUSTRIELLE}

L'émulsion de $\beta$-carotène une fois mise au point, il devenait possible de l'utiliser dans des fabrications industrielles de beurre.

Conditions expérimentales : Des essais ont été effectués dans une beurrerie équipée pour la fabrication continue du beurre. La crème à 38 p. 100 de matière grasse était maturée dans deux tanks de 25001 :

- un tank témoin,

- un tank d'expérience où la crème était additionnée d'émulsion de $\beta$-carotène.

Pour cette addition une quantité exactement mesurée d'émulsion à 2 p. 100 de $\beta$-carotène est mélangée à 31 de crème, puis le mélange est versé dans la crème du tank d'expérience.

Au cours de ces essais, on a ajouté à la crème des doses croissantes de $\beta$-carotène. Le beurre obtenu dans chacune de ces fabrications était de couleur homogène. Aucune marbrure n'a été constatée après la fabrication ou pendant la conservation du produit fini. La phase aqueuse du babeurre n'est pas colorée.

\section{TABLEAU 3}

Evolution des teneurs en carotène des beurres pendant la conservation à $+4^{\circ} \mathrm{C}$ (plaquette $250 \mathrm{~g}$ - emballage papier aluminium)

Teneurs en mg p. $100 \mathrm{~g}$ de beurre

\begin{tabular}{c|c|c|c}
\hline \multirow{2}{*}{ Echantillon } & \multicolumn{3}{|c}{ Durée de conservation } \\
\cline { 2 - 4 } & 0 & 2 mois & 4 mois \\
\hline$A_{2}$ & 0,349 & 0,354 & 0,350 \\
$C_{2}$ & 0,543 & 0,537 & 0,535 \\
$\mathrm{~F}_{2}$ & 0,537 & 0,540 & 0,530 \\
\hline
\end{tabular}

Il serait superflu de reproduire les résultats de tous les essais industriels effectués. Ils ont tous permis des constatations identiques à celles qui découlent de l'essai $\mathrm{n}^{\circ} 7$ décrit ci-dessous. 
ESSAI $\mathrm{N}^{\circ} 7$

Dans 2500 kg de crème à 38 p. 100 de matière grasse, on incorpore une quantité d'émulsion correspondant à un ajout de $0,284 \mathrm{mg}$ de $\beta$-carotène par $100 \mathrm{~g}$ de matière grasse.

Les analyses effectuées sur les beurres témoins et d'expérience donnent les résultats suivants :

Teneurs en $\beta$-carotène (en $\mathrm{mg}$ dans $100 \mathrm{~g}$ de matière grasse)

$\begin{array}{lllllll}\text { Beurre témoin .. . . . } & 0,426 & 0,415 & & & & \text { Moyenne } \\ \text { Beurre d'expérience } & \ldots & 0,662 & 0,673 & 0,666 & 0,655 & 0,664\end{array}$

En tenant compte des moyennes de ces résultats, on peut calculer la quantité de $\beta$-carotène ajouté qui est retrouvée dans le beurre soit :

$0,664-0,420=0,244 \mathrm{mg}$ pour $100 \mathrm{~g}$ de matière grasse. Le pourcentage de $\beta$-carotène ajouté restant dans le beurre est voisin de 86 p. 100.

Il est intéressant de noter l'homogénéité des teneurs en $\beta$-carotène de différents échantillons d'une même fabrication. Ceci témoigne de la bonne dispersion de l'émulsion dans la crème.

\section{V. - Méthodologie de la normalisation}

Grâce à l'étroite corrélation entre la coloration du beurre et la teneur en $\beta$-carotène, la connaissance de l'une de ces deux caractéristiques suffit à déterminer la couleur du beurre de référence et, en fonction de la teinte désirée dans le produit fini, de calculer la complémentation nécessaire en pigment.

La détermination de la couleur demande la mise au point d'une gamme colorimétrique à laquelle seront comparées des fabrications expérimentales. La teneur en $\beta$-carotène, eu égard aux simplifications possibles de la méthode d'analyse, peut être déterminée assez facilement et rapidement.

Ainsi pour une crème à 33 p. 100 de matière grasse contenant $1,2 \mathrm{ppm}$ de $\beta$-carotène, le beurre obtenu aurait une teneur de $3 \mathrm{ppm}$ en pigment. Pour normaliser la couleur à $7 \mathrm{ppm}$, il faudrait ajouter $80 \mathrm{~g}$ d'émulsion à 2 p. 100 dans 10001 de crème, permettant d'obtenir $400 \mathrm{~kg}$ de beurre d'une teinte conforme à celle définie au départ.

Pour effectuer correctement la normalisation il est nécessaire :

$1^{\circ}$ de déterminer la teinte désirée dans le produit fini, teinte correspondant à un taux défini de $\beta$-carotène soit $\mathrm{R} \mathrm{ppm}$,

$2^{\circ}$ de connaître pour la crème ses teneurs G p. 100 en matière grasse et $\mathrm{K}$ en ppm de $\beta$-carotène,

$5^{\circ}$ de calculer $\mathrm{S}$, quantité en grammes d'émulsion à 2 p. 100 de $\beta$-carotène à ajouter par tonne de crème au moyen de la formule :

$$
\mathrm{S}=\left(\frac{\mathrm{RG}}{82}-\mathrm{K}\right) \times 50
$$




\section{Conclusion}

Les résultats obtenus au cours des essais industriels montrent que la coloration du beurre à l'aide de $\beta$-carotène est technologiquement possible et simple à réaliser.

La mise au point d'une forme de $\beta$-carotène à teneur garantie, jointe à une méthode simple de contrôle de la matière première utilisée, telles qu'elles sont définies par la présente étude, permettent une normalisation aisée et rigoureuse de la couleur du produit fini.

\section{Remerciements}

Nous adressons nos plus vifs remerciements à la Laiterie Reneau à Cuincy-les-Douai qui nous a permis d'effectuer les essais industriels.

\section{Rés u m é}

Lors d'études précédentes, on a constaté d'importantes variations du taux de $\beta$-carotène dans les beurres, entrainant une grande disparité de couleur.

On a cherché, à l'aide de $\beta$-carotène de synthèse, à normaliser la teinte du produit fini, en estimant que la préférence des consommateurs correspond à des beurres contenant environ $7 \mathrm{ppm}$ de pigment.

Une émulsion contenant 2 p. 100 de $\beta$-carotène a été mise au point. D'un emploi facile, elle permet d'obtenir des beurres de couleur homogène. Sa conservation à $5^{\circ} \mathrm{C}$ est bonne. Lors de l'utilisation, les pertes dans le babeurre sont négligeables. Ceci a été vérifié tant au laboratoire que lors d'essais industriels.

La détermination du taux de matière grasse et de la teneur en $\beta$-carotène permet, à l'aide d'une formule simple, de calculer la quantité d'émulsion de $\beta$-carotène nécessaire pour obtenir la teneur en pigment correspondant à la teinte désirée du produit fini.

\section{S u m m a ry}

A precedent study has shown the importance of the variability of $\beta$-carotene content in butter. This involves a large dispersion in the colour of this product. We have looked after the standardization of colour using synthetic $\beta$-carotene. The precedent study showed that the consumer prefers butter containing about $7 \mathrm{ppm}$ of $\beta$-carotene.

An experimental 2 p. 100 B-carotene emulsion was made. Easy to use, it allows the manufacturing of butter with homogenous colour even in continous process. During storage at $5^{\circ} \mathrm{C}$ there is no alteration of its properties especially $\beta$-carotene content.

No losses of $\beta$-carotene in buttermilk could be measured, neither in laboratory experiments, nor in industrial buttermaking. 
With the determination of $\beta$-carotene and fat contents of cream, a simple formula aliows to calculate the quantity of 2 p. $100 \beta$-carotene emulsion to be added in order to obtain the amount of $\beta$-carotene in the final product, corresponding to the wished colour.

\section{Bibliographie}

Aust (H.) (1955). - Die Färbung der Butter mit synthetischen $\beta$-Carotin. Milchwissenschaftliche Berichte, $5, \mathrm{n}^{\circ} 2$.

Casalis (J)., Chardon (Y.) Luquet (F. M.), Mainguy (P.) et Yver (M.) (1972). Sur la relation entre la teneur en $\beta$-carotène et la couleur des beurres français. Le Lait, janvier-février, $\mathrm{n}^{\circ}$ 511-512, p. 28-42.

GLOVER (J.) (1960). - The conversion of $\beta$-carotene into vitamin A. Vitamins and Hormones, 18, 371-386.

Hugot (D.) et CAUSERET (J.) (1966). - Variations saisonnières de l'activité vitaminique A de beurres produits dans diverses régions de France. $17^{\mathrm{e}}$ Congrès Int. Laiterie, C 185-C 191.

IsLeR (O.), GutManN (H.) und Solms (U.) (1971). - Carotenoids (932 p.), Birkhaüser Verlag - Basel.

KLAUI (H.) (1968). - The use of synthetic Carotenoids for colouring of foods, Inst. of Food Science and Techn. Proceedings Special colour symposium issue, vol. $\mathrm{I}, \mathrm{n}^{\circ} 5$.

LucK (H.) and Novello (J. C.) (1970). - Measuring and Standardising the colour of butter. South African Journal Dairy Tech., 2, n ${ }^{\circ}$ 2, p. 91-96.

LucK (H.), Novello (J. C.) and VAN ZYL (D. J.) (1970). - Colouring of butter by means of annato and $\beta$-carotene. South Afr. J. of Dairy Tech., vol. 1, $\mathrm{n}^{\circ} 1$.

RIEL (R. R.) and JoHns (C. K.) (1957). - The use of synthetic $\beta$-carotene for colouring butter. J. of Dairy Science, 40, $\mathrm{n}^{\circ}$ 2, 192-199.

Searles (S. K.) and Armstrong (J. G.) (1970). - Vitamin A, vitamin E and Carotene contents of Alberta butter. J. of Dairy Science, 53, $\mathrm{n}^{\circ} 2,150-154$.

STüssi (D.) (1957). - Uber Versuche mit $\beta$-Carotin synth. "Roche" zur Butter Färbung. Schweizerische Milchzeitung, $\mathrm{n}^{\circ}$ 99-101, Wiss. Beilage, $\mathrm{n}^{\circ}$ 54-55.

YVER (M.) et MAINGUY (P.) (1971). - Les caroténoïdes, nouveaux agents colorants mis à la disposition des industries alimentaires. Cycle conférences CTU, Certains additifs chimiques, p. 31-41.

ZBINDEN (G.) und StüDER (A.) (1958). - Tiere experimentelle Untersuchungen über die chronische Verträglichkeit von $\beta$-carotin, Lycopin, 7, 7'-dihydro- $\beta$ carotin und Bixin. Zeitschrift für Lebensmittel-Untersuchung und-Forschung, $108, \mathrm{n}^{\circ} 2,113-134$. 